\title{
EXCHANGE RATE FORECASTING USING FUZZY TIME SERIES-MARKOV CHAIN
}

\section{LIM XIN HUI AND BINYAMIN YUSOFF*}

Faculty of Ocean Engineering Technology and Informatics, Universiti Malaysia Terengganu, 21030 Kuala Nerus, Terengganu, Malaysia.

*Corresponding author: binyamin@umt.edu.my

http://doi.org/10.46754/umtjur.2021.07.019

\begin{abstract}
Exchange rate forecasting plays an important role in financial management. However, it is a complex process with high nonlinearity and data irregularity. Moreover, the forecasting of exchange rate is highly involved with imprecise and uncertain data. Analysis of forecasting models which corresponds to the exchange rate has always experienced fluctuations. Therefore, exchange rate forecasting becomes a challenging task in finance. Several studies have shown that stand-alone forecasting models such as time series, fuzzy time series, and Markov chain have their own drawbacks and are not successful enough in forecasting accurately. In this study, we propose a hybrid model of fuzzy time series-Markov chain to forecast the future exchange rate. Fuzzy time series-Markov chain is a combination of the classic fuzzy time series model with Markov chain model used to analyse a set of time series data. The main motivation for this study is to improve the accuracy in exchange rate forecasting. The selected currencies are Malaysian Ringgit (MYR) and Singapore Dollar (SGD). The proposed model was then evaluated by the Mean Absolute Percentage Error (MAPE) performance metric to test the robustness of the model. Lastly, a comparison between the proposed model and fuzzy time series model was conducted with respect to the MAPE. The results showed that the MAPE value for fuzzy time series-Markov chain was $0.9895 \%$ which fell under the criterion of highly accurate forecasting. Meanwhile, the MAPE value for fuzzy time series was $3.4306 \%$. Thus, the forecasting performance of the proposed model was better than the fuzzy time series model. This study reveals the potential benefits of the proposed model as a highly accurate forecasting model.
\end{abstract}

Keywords: Exchange rate forecasting, fuzzy set theory, time series, fuzzy time series, markov chain, fuzzy time series Markov chain, MAPE

\section{Introduction}

Exchange rate is an important consideration in the globalized market. Hence, forecasting of the exchange rate plays a crucial role in international financial management. It provides information on the current states and changes in exchange rate. A successful prediction of future exchange rate can yield a significant profit, assisting company management and investors to make an informed decision in financial decisionmaking process.

Forecasting involves making an informed prediction to determine future trends. It is the most essential method in financial management. In general, there are two types of forecasting methods reported in the literature: qualitative and quantitative models. The qualitative model is based on non-statistical approaches such as subjective judgments or expert opinion, and personal experiences while the quantitative model relies on objective and statistical approach in forecasting. Some of the examples of forecasting models are market survey, simple mean, simple moving average, exponential smoothing, Markov chain, linear regression, and multiple regression (Reid \& Sanders, 2010).

The time series models are one of the most important elements in forecasting (Islam \& Hossain, 2020). Time series forecasting is a method that collects and analyses previous 
observations or experiences of the same system to develop a model describing an underlying relationship. It uses time as an independent variable in the relationship. There are two types of time series: discrete and continuous. The measurements of time series models are usually daily, weekly, monthly, quarterly, annually or any suitable time intervals (Chatfield, 2000).

Time series model helps us to analyse past behaviour and compare the predicted result to the past performance. However, the time series model does not always meet a satisfactory result in exchange rate forecasting. The exchange rate is influenced by some other parameters including the psychological and sociological factors that cannot easily be measured and are very noisy. It tends to fluctuate unexpectedly. Besides, time series models are linear and have chaotic behaviours (Erdogan \& Goksu, 2014). It is unable to capture the nonlinear relationship in the movement of the exchange rate.

Markov chain, as proposed by Andrey Andreyevich Markov, is another widely used forecasting model in finance (Seneta, 2006). It is a stochastic process with 'no-after effect' property and its state space is discrete. Markov model only considers the characteristic of the historical data and predicts changes by using transition probability. Because of its 'no-after effect' property, it is more effective under the market mechanism (Zhang \& Zhang, 2009). Since the Markov chain is a probability forecasting method, it only provides the predicted result in probability state rather than a proper value. Moreover, it also requires the data to be accurate and precise.

Despite the importance of time series and Markov chain models for forecasting, other issues related to these models have been reported in the literature. These include uncertainty problems due to ambiguity or imprecision. The real application of these models is usually accompanied by this type of uncertainty. Hence, the models become unstable and unreliable when uncertainty exists. Zadeh (1965) introduced a fuzzy set theory to cope with this type of uncertainty. The linguistic variable is an important concept in fuzzy set theory, transforming values into words or sentences in the natural or artificial language (Zadeh, 1973). It collects elements into similar groups so that we can deal with less precise data and handle more complex systems. Fuzzy logic, on the other hand, is based on fuzzy set concept and is a flexible model to deal with human reasoning and decision making. It can add more functionality and allows the imprecise data in the model (Meyer \& Zimmermann, 2011).

For the time series forecasting, if the historical data are insufficient or associated with linguistic values, vagueness and imprecision, the applicability of these models will be affected. To cope with this uncertainty in the data, Song and Chissom $(1993$; 1994) proposed a fuzzy time series model to handle the forecasting problems using fuzzy logic. Later, the simplification of this model was proposed by Chen (1996). This model has shown its effectiveness in the foreign exchange problem (Boiroju et al., 2011). It also has more capability in handling the nonlinear relationships. Although the uncertainty represented by the linguistic terms increases the interpretability of the data, but there are still some limitations to the fuzzy time series model. First, the temporal patterns were defined by rigid regions that were hard to adjust when there is noise exist. Next, it requires complex computational skills and a lack of stability (Tayal, 2011).

Similarly, considering that the uncertainty exists in realistic states, the Markov chain may be less effective to deal with the complex task. Hence the fuzzy version of Markov chain has been proposed in the literature to tackle this problem (Li \& Xiu, 2014; Uzun \& Kiral, 2017). Avrachenkov and Sanchez (2002) have shown that the fuzzy Markov chain is a robust system with respect to small perturbations of the transition matrix, which is not the case for the classical probabilistic Markov chains.

As stated above, the movement of exchange rate is affected by various factors. Therefore, no single method can take those considerations into account and predict the changes in exchange 
rate accurately. Fortunately, we can combine different methods to improve the performance and reduce the forecasted error of the standalone models. For example, Tsaur (2012) has proposed a fuzzy time series-Markov chain model to overcome the above highlighted problems. The efficacy of the proposed model has been shown in forecasting the exchange rate between Taiwan and US dollar (Tsaur, 2012). Thus, with the hybrid model, it is implied that the more the information pertaining to the system dynamics is induced, the better the forecasting will be. Therefore, in this study, we propose to apply the fuzzy time series-Markov chain model to forecast the exchange rate between the Malaysian Ringgit (MYR) and Singapore Dollar (SGD) based on monthly time series data.

Various performance measures have been proposed to estimate the forecast accuracy and to test the robustness of the models (Sahin et $a l ., 2014)$. These are known as performance metrics. The examples of these measures are mean forecast error (MFE), mean percentage error (MPE), mean absolute percentage error (MAPE), mean squared error (MSE), and sum of squared error (SSE), among others (Adhikari \& Agrawal, 2013). In this study, MAPE is used to test the robustness of the proposed model. This selection is due to its advantages of scaleindependency and interpretability.

The remainder of the paper is organized as follows: Section 2 is devoted to the preliminaries where the definitions and basic concepts of fuzzy set theory, fuzzy time series, and Markov chain are provided. Section 3 introduces the methodology of the proposed model: fuzzy time series-Markov chain model and MAPE performance metric. In Section 4, the case study of forecasting the exchange rate between MYR and SGD is put forward. Furthermore, Section 5 presents the results and discussion of this study. Finally, some concluding remarks are given in Section 6.

\section{Preliminaries}

\section{Fuzzy Set Theory}

Fuzzy set theory states that a certain element may belong or simultaneously not belong to the same set of certain degree. This theory can be mathematically defined as the following:

Definition 1 (Zadeh, 1965): A fuzzy set in a universe $X$ is a set that is defined as:

$$
\tilde{A}=\left\{\left(\mu_{\tilde{A}}(x), x\right) \mid x \in X\right\},
$$

where $\mu_{\tilde{A}}(x) \in[0,1]$ represents the degree of membership of element $x$ in $\tilde{A}$.

Definition 2 (Chang \& Zadeh, 1972): A fuzzy number $\tilde{A}=\left(a_{1}, a_{2}, a_{3}\right)$ where $a_{1}<a_{2}<a_{3}$ is called triangular fuzzy number if its membership function is given by:

$$
\mu_{\tilde{A}}(x)=\left\{\begin{array}{cl}
\frac{x-a_{1}}{a_{2}-a_{1}}, & \text { if } a_{1} \leq x \leq a_{2} \\
\frac{a_{3}-x}{a_{3}-a_{2}}, & \text { if } a_{2} \leq x \leq a_{3} \\
0, & \text { otherwise. }
\end{array}\right.
$$

The other types of membership functions are trapezoidal membership function, Gaussian membership function, sigmoid membership function, to name a few (Madi, et al., 2020; Ali, et al., 2015).

\section{Fuzzy Time Series}

In the following, the definitions and basic concept of fuzzy time series are presented. Song and Chissom (1993) first established the definitions of fuzzy time series. Then, the simplification of this model was proposed by Chen (1996). The definitions presented here are merely based on Chen (1996).

Definition 3 (Chen, 1996): Let $U$ be the universal discourse with $U=\left(u_{1}, u_{2}, \ldots, u_{n}\right)$. A fuzzy set $A_{i}$ in the universal discourse, $U$ is defined as:

$$
A_{i}=\frac{f_{A_{i}}\left(u_{1}\right)}{u_{1}}+\frac{f_{A_{i}}\left(u_{2}\right)}{u_{2}}+\cdots+\frac{f_{A_{i}}\left(u_{n}\right)}{u_{n}},
$$


where $f_{A_{i}}$ represents the membership function of fuzzy set $A_{i}, u_{j}$ is an element in $A_{i}$ and $f_{A_{i}}\left(u_{j}\right)$ is the degree of membership of $u_{j}, f_{A_{i}}$ $\left(u_{j}\right) \in[0,1]$ and $1 \leq j \leq n$.

Definition 4 (Chen, 1996): Suppose $Y(t-1)=A_{i}$ and $Y(t)=A_{j}$ where $A_{i}$ and $A_{j}$ are fuzzy set, the fuzzy logical relationship between $Y(t-1)$ and $Y(t)$ can be expressed as $A_{i} \rightarrow A_{j}$ where,

\section{$Y(t-1):$ collected data at time $t-1$}

\section{$Y(t)$ : collected data at time $t$}

$A_{i}:$ current set of the fuzzy logical relationship

$A_{j}$ : next state of the fuzzy logical relationship

\section{Markov Chain}

Markov Chain is a stochastic process with Markov property. It is a time series model with discrete state (He \& Jiang, 2018).

Definition 5 (Adesokan et al., 2017): A Markov chain is a collection of random variables (where the index runs through $0,1, \ldots$ ) having the property that, given the present, the future is conditionally independent of the past. Mathematically, it can be presented as:

$P\left(X_{i}=j \mid X_{0}=i_{0}, X_{1}=i_{1}, \ldots, X_{t-1}=i_{t-1}\right)=P\left(X_{i}=j \mid X_{t-1}=i_{t-1}\right)$.

Definition 6 (Ching \& Ng, 2006): State $i$ is said to be reachable from state $j$ if $P_{i j}^{(n)}>0$ and for some $n>0$. This means that starting from state $j$, it is possible to enter state $i$ in finite number of transitions.

Definition 7 (Ching \& Ng, 2006): State $i$ and state $i$ are said to communicate if state and state $j$ are reachable from each other.

\section{Methodology}

\section{Fuzzy Time Series-Markov Chain}

Tsaur (2012) first developed this method by using the Song and Chissom $(1993 ; 1994)$ and Chen (1996) concepts. The flowchart of fuzzy time series-Markov chain is presented in Figure 1. Then, the algorithm of fuzzy time seriesMarkov chain is shown as the following:

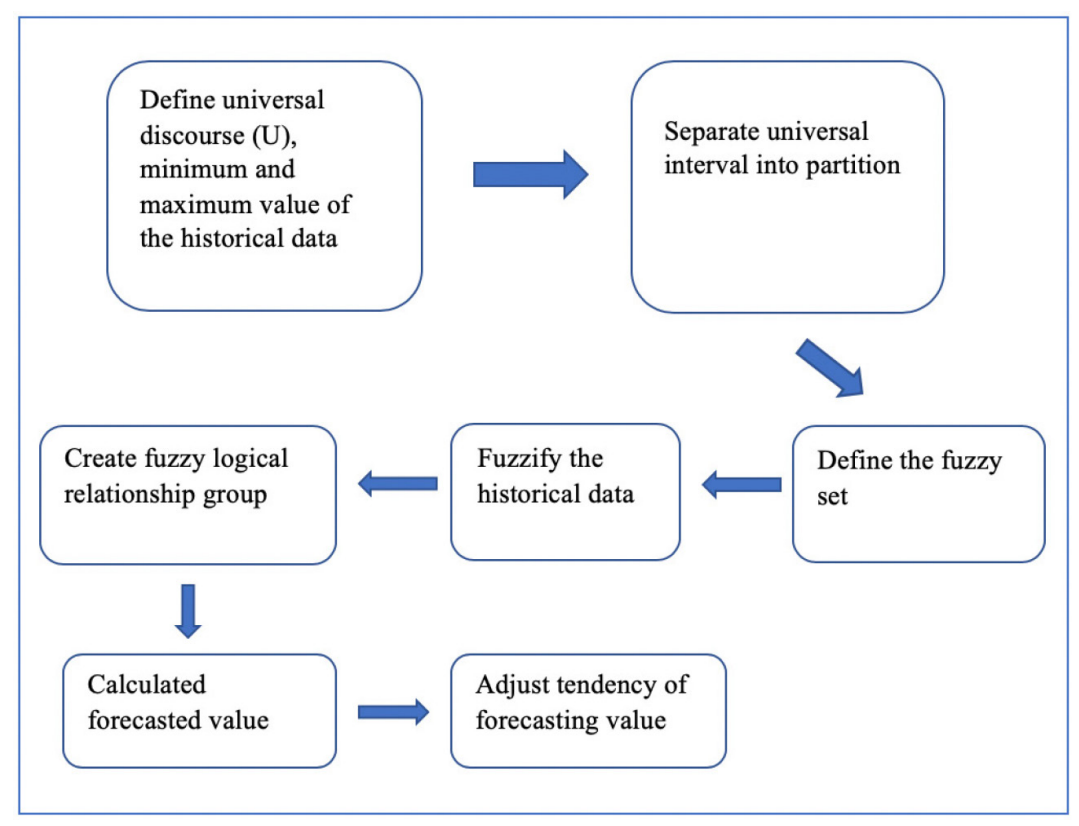

Figure 1: Flowchart of fuzzy time series-Markov chain. NB: Calculate forecasted value 
Step 1: Define the universal discourse $U$, minimum data $\left(D_{\mathrm{min}}\right)$ and maximum data $\left(D_{\max }\right)$ for the historical data. The universal discourse is defined as:

$$
U=\left[D_{\min }-D_{1}, D_{\max }+D_{2}\right] .
$$

Step 2: Separate $U$ into several equal intervals. The universal of discourse is then divided into 7 equal-length intervals with $l=0.1003$.

Step 3: Define the fuzzy set using Eq. (6):

$$
\begin{gathered}
A_{1}=\frac{a_{11}}{u_{1}}+\frac{a_{12}}{u_{2}}+\cdots+\frac{a_{1 n}}{u_{n}}, \\
A_{2}=\frac{a_{21}}{u_{1}}+\frac{a_{22}}{u_{2}}+\cdots+\frac{a_{2 n}}{u_{n}}, \\
\vdots \\
A_{k}=\frac{a_{k 1}}{u_{1}}+\frac{a_{k 2}}{u_{2}} \cdots+\frac{a_{k n}}{u_{n}},
\end{gathered}
$$

where $A_{i}, i=1, \ldots, k$ are the fuzzy sets which are represented as linguistic values of the linguistic variables.

Step 4: Fuzzify the historical data to fuzzy set $A_{i}$.

Step 5: Create fuzzy logical relationship group.

Step 6: Calculate forecasting value, $f(t)$ according to the rules.

Rule 1: If $Y(t-1)=A_{j}$ and it is one-to-one fuzzy logical relationship, $A_{j} \rightarrow A_{k}$, the forecasted future value, $f(t)$ is calculated by using Eq. (7).

$$
f(t)=m_{k}
$$

Rule 2: If $Y(t-1)=A_{j}$ and it is one-tomany fuzzy logical relationship, for example $A_{j} \rightarrow A_{1}, A_{2}, \ldots, A_{n} j=1,2, \ldots, n$, the forecasted future value, $f(t)$ is:

$$
\begin{gathered}
F(t)=m_{1} P_{j 1}+m_{2} P_{j 2}+\cdots+m_{j-1} P_{j(j-1)}+Y(t-1) \\
P_{j j}+m_{j+1} P_{j(j+1)}+\cdots+m_{n} P_{j n},
\end{gathered}
$$

where,

$m_{i}:$ midpoint of interval $u_{i}$

$P_{j n}:$ probability from state $j$ to state $n$

Step 7: Adjust the tendency of the forecasting value according to the rules.

Rule 1: If $A_{i}$ communicates with $A_{i}$ and makes an increasing transition, where $Y(t-1)=$ $A_{i}, Y(t)=A_{j},\left(A_{i} \rightarrow A_{j} i>j\right)$, then the adjusted trend value will be:

$$
D_{t 1}=\frac{l}{2} .
$$

Rule 2: If $A_{i}$ communicates with $A_{i}$ and makes a decreasing transition, where $Y(t-1)=$ $A_{i}, Y(t), A_{j}\left(A_{i} \rightarrow A_{j}, i>j\right)$, then the adjusted trend value will be:

$$
D_{t 1}=-\frac{l}{2}
$$

Rule 3: If $Y(t-1)=A_{i}$ and makes a jumpforward transition at time $t$ where $Y(t)=A_{i+s}$, $(1 \leq s \leq n-i)$, then the adjusted trend value will be:

$$
D_{t 2}=\frac{l}{2}(s)
$$

Rule 4: If $Y(t-1)=A_{i}$ and makes a jumpbackward transition at time $t$ where $Y(t)=A_{i-v}$, $(1 \leq v \leq i)$ then the adjusted trend value will be:

$$
D_{t 2}=-\frac{l}{2}(v)
$$

Step 8: Calculate the adjusted forecasting values.

$$
F^{\prime}(t)=F(t) \pm D_{t 1} \pm D_{t 2}
$$

\section{Performance Evaluation of Model}

The performance of the proposed model is evaluated by using the following Mean Absolute Percentage Error (MAPE) formula:

$$
M A P E=\frac{1}{n} \sum_{t=1}^{n} \frac{\left|Y(t)-F^{\prime}(t)\right|}{Y(t)} \times 100 \% .
$$




\section{Analysis on Case Study}

In this study, the historical data of exchange rate between MYR and SGD from year 2015 to year 2019 were collected from official website of Bank Simpanan Nasional (www.bnm.gov.my). The proposed model fuzzy time series-Markov chain is used to forecast the exchange rate by using Microsoft Excel.

Step 1: The universal discourse, $U$ in this study is defined as $U=[2.4570,3.1590]$.

Step 2: The universal of discourse is then divided into 7 equal-length intervals with $l=$ 0.1003 . Then, the interval is defined as below:

$$
\begin{aligned}
& u_{1}=[2.4570,2.5572], \\
& u_{2}=[2.5573,2.6575], \\
& u_{3}=[2.6576,2.7578], \\
& u_{4}=[2.7579,2.8581], \\
& u_{5}=[2.8582,2.9583], \\
& u_{6}=[2.9584,3.0586], \\
& u_{7}=[3.0587,3.1589] .
\end{aligned}
$$

Step 3: Consider the forecasting of interval in triangular membership function, the fuzzy sets are defined as:

$$
\begin{aligned}
& A_{1}=\frac{1}{u_{1}}+\frac{0.5}{u_{2}}+\frac{0}{u_{3}}+\frac{0}{u_{4}}+\frac{0}{u_{5}}+\frac{0}{u_{6}}+\frac{0}{u_{7}}, \\
& A_{2}=\frac{0.5}{u_{1}}+\frac{1}{u_{2}}+\frac{0.5}{u_{3}}+\frac{0}{u_{4}}+\frac{0}{u_{5}}+\frac{0}{u_{6}}+\frac{0}{u_{7}}, \\
& A_{3}=\frac{0}{u_{1}}+\frac{0.5}{u_{2}}+\frac{1}{u_{3}}+\frac{0.5}{u_{4}}+\frac{0}{u_{5}}+\frac{0}{u_{6}}+\frac{0}{u_{7}}, \\
& A_{4}=\frac{0}{u_{1}}+\frac{0}{u_{2}}+\frac{0.5}{u_{3}}+\frac{1}{u_{4}}+\frac{0.5}{u_{5}}+\frac{0}{u_{6}}+\frac{0}{u_{7}}, \\
& A_{5}=\frac{0}{u_{1}}+\frac{0}{u_{2}}+\frac{0}{u_{3}}+\frac{0.5}{u_{4}}+\frac{1}{u_{5}}+\frac{0.5}{u_{6}}+\frac{0}{u_{7}}, \\
& A_{6}=\frac{0}{u_{1}}+\frac{0}{u_{2}}+\frac{0}{u_{3}}+\frac{0}{u_{4}}+\frac{0.5}{u_{5}}+\frac{1}{u_{6}}+\frac{0.5}{u_{7}}, \\
& A_{7}=\frac{0}{u_{1}}+\frac{0}{u_{2}}+\frac{0}{u_{3}}+\frac{0}{u_{4}}+\frac{0}{u_{5}}+\frac{0.5}{u_{6}}+\frac{1}{u_{7}} .
\end{aligned}
$$

Step 4: The fuzzified exchange rate is matched with its equivalent fuzzy sets. The result is shown in Table 1.

Table 1: The Fuzzification of Data

\begin{tabular}{cccccc}
\hline Month & Exchange rate & Fuzzy data & Month & Exchange rate & Fuzzy data \\
\hline Jan-15 & 2.6792 & $A_{3}$ & Jul-17 & 3.1282 & $A_{7}$ \\
Feb-15 & 2.6524 & $A_{2}$ & Aug-17 & 3.1482 & $A_{7}$ \\
Mar-15 & 2.6726 & $A_{3}$ & Sep-17 & 3.1193 & $A_{7}$ \\
Apr-15 & 2.6923 & $A_{3}$ & Oct-17 & 3.1085 & $A_{7}$ \\
May-15 & 2.6999 & $A_{3}$ & Nov-17 & 3.0769 & $A_{7}$ \\
Jun-15 & 2.7781 & $A_{4}$ & Dec-17 & 3.0284 & $A_{6}$ \\
Jul-15 & 2.7934 & $A_{4}$ & Jan-18 & 2.9925 & $A_{6}$ \\
Aug-15 & 2.9042 & $A_{5}$ & Feb-18 & 2.9635 & $A_{6}$ \\
Sep-15 & 3.0458 & $A_{6}$ & Mar-18 & 2.9690 & $A_{6}$ \\
Oct-15 & 3.0432 & $A_{6}$ & Apr-18 & 2.9555 & $A_{5}$ \\
Nov-15 & 3.0506 & $A_{6}$ & May-18 & 2.9622 & $A_{6}$ \\
Dec-15 & 3.0393 & $A_{6}$ & Jun-18 & 2.9687 & $A_{6}$ \\
Jan-16 & 3.0323 & $A_{6}$ & Jul-18 & 2.9704 & $A_{6}$ \\
Feb-16 & 2.9792 & $A_{6}$ & Aug-18 & 2.9896 & $A_{6}$ \\
\hline
\end{tabular}




\begin{tabular}{cccccc}
\hline Mar-16 & 2.9655 & $A_{6}$ & Sep-18 & 3.0209 & $A_{6}$ \\
Apr-16 & 2.8904 & $A_{5}$ & Oct-18 & 3.0154 & $A_{6}$ \\
May-16 & 2.9535 & $A_{5}$ & Nov-18 & 3.0433 & $A_{6}$ \\
Jun-16 & 3.0140 & $A_{6}$ & Dec-18 & 3.0453 & $A_{6}$ \\
Jul-16 & 2.9755 & $A_{6}$ & Jan-19 & 3.0350 & $A_{6}$ \\
Aug-16 & 2.9901 & $A_{6}$ & Feb-19 & 3.0092 & $A_{6}$ \\
Sep-16 & 3.0233 & $A_{6}$ & Mar-19 & 3.0125 & $A_{6}$ \\
Oct-16 & 3.0177 & $A_{6}$ & Apr-19 & 3.0340 & $A_{6}$ \\
Nov-16 & 3.0737 & $A_{7}$ & May-19 & 3.0438 & $A_{6}$ \\
Dec-16 & 3.1070 & $A_{7}$ & Jun-19 & 3.0540 & $A_{6}$ \\
Jan-17 & 3.1214 & $A_{7}$ & Jul-19 & 3.0322 & $A_{6}$ \\
Feb-17 & 3.1422 & $A_{7}$ & Aug-19 & 3.0242 & $A_{6}$ \\
Mar-17 & 3.1583 & $A_{7}$ & Sep-19 & 3.0329 & $A_{6}$ \\
Apr-17 & 3.1521 & $A_{7}$ & Oct-19 & 3.0542 & $A_{6}$ \\
May-17 & 3.0933 & $A_{7}$ & Nov-19 & 3.0545 & $A_{6}$ \\
Jun-17 & 3.0909 & $A_{7}$ & Dec-19 & 2.4573 & $A_{1}$
\end{tabular}

Step 5: The fuzzy logical relationship in this study is determined and shown in Figure 2

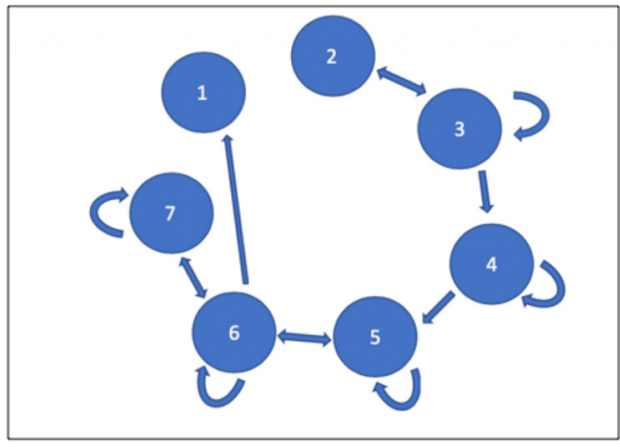

Figure 2: Transition process of the exchange rate

Step 6: The forecasting value, $F(t)$ is calculated according to the rules. The forecasting value for Feb-15 in this study is shown as below.

$$
\begin{aligned}
F(F e b-15) & =\frac{1}{4} m_{2}+\frac{1}{2}(F(t-1))+\frac{1}{4}\left(m_{4}\right) \\
& =\frac{1}{4}(2.6074)+\frac{1}{2}(F(t-1))+\frac{1}{4}(2.8080) \\
& =\frac{1}{4}(2.6074)+\frac{1}{2}(2.6792)+\frac{1}{4}(2.8080) \\
& =2.6934 .
\end{aligned}
$$

Step 7: The tendency of the forecasting value is adjusted according to the rules. For example, the adjustment for Feb-15 is obtained as follows:

$$
\begin{aligned}
\text { Adjustment for Feb }-15 & =-D_{t 1} \\
& =-\frac{l}{2} \\
& =-\frac{0.1003}{2} \\
& =-0.0501 .
\end{aligned}
$$


Step 8: The adjusted forecasting values, $F^{\prime}(t)$ are Results and Discussion obtained and presented in Table 2.

The result of the forecasting value using fuzzy time series-Markov chain is shown in Table 2.

Table 2: Forecasting value of the exchange rate between MYR and SGD

\begin{tabular}{|c|c|c|c|c|c|}
\hline Month & Actual Exchange rate & $F^{\prime}(t)$ & Month & Actual Exchange rate & $F^{\prime}(t)$ \\
\hline Jan-15 & 2.6792 & & Jul-17 & 3.1282 & 3.0845 \\
\hline Feb-15 & 2.6524 & 2.6433 & Aug-17 & 3.1482 & 3.1190 \\
\hline Mar-15 & 2.6726 & 2.7077 & Sep-17 & 3.1193 & 3.1375 \\
\hline Apr-15 & 2.6923 & 2.6901 & Oct-17 & 3.1085 & 3.1108 \\
\hline May-15 & 2.6999 & 2.7000 & Nov-17 & 3.0769 & 3.1008 \\
\hline Jun-15 & 2.7781 & 2.7539 & Dec-17 & 3.0284 & 3.0215 \\
\hline Jul-15 & 2.7934 & 2.8432 & Jan-18 & 2.9925 & 3.0089 \\
\hline Aug-15 & 2.9042 & 2.9010 & Feb-18 & 2.9635 & 2.9771 \\
\hline Sep-15 & 3.0458 & 3.0326 & Mar-18 & 2.9690 & 2.9515 \\
\hline Oct-15 & 3.0432 & 3.0244 & Apr-18 & 2.9555 & 2.9062 \\
\hline Nov-15 & 3.0506 & 3.0220 & May-18 & 2.9622 & 3.0454 \\
\hline Dec-15 & 3.0393 & 3.0286 & Jun-18 & 2.9687 & 2.9503 \\
\hline Jan-16 & 3.0323 & 3.0186 & Jul-18 & 2.9704 & 2.9561 \\
\hline Feb-16 & 2.9792 & 3.0124 & Aug-18 & 2.9896 & 2.9575 \\
\hline Mar-16 & 2.9655 & 2.9653 & Sep-18 & 3.0209 & 2.9746 \\
\hline Apr-16 & 2.8904 & 2.9031 & Oct-18 & 3.0154 & 3.0023 \\
\hline May-16 & 2.9535 & 2.9790 & Nov-18 & 3.0433 & 2.9974 \\
\hline Jun-16 & 3.0140 & 3.0449 & Dec-18 & 3.0453 & 3.0222 \\
\hline Jul-16 & 2.9755 & 2.9962 & Jan-19 & 3.0350 & 3.0239 \\
\hline Aug-16 & 2.9901 & 2.9620 & Feb-19 & 3.0092 & 3.0148 \\
\hline Sep-16 & 3.0233 & 2.9750 & Mar-19 & 3.0125 & 2.9919 \\
\hline Oct-16 & 3.0177 & 3.0044 & Apr-19 & 3.0340 & 2.9948 \\
\hline Nov-16 & 3.0737 & 3.0496 & May-19 & 3.0438 & 3.0139 \\
\hline Dec-16 & 3.1070 & 3.0687 & Jun-19 & 3.0540 & 3.0225 \\
\hline Jan-17 & 3.1214 & 3.0994 & Jul-19 & 3.0322 & 3.0316 \\
\hline Feb-17 & 3.1422 & 3.1127 & Aug-19 & 3.0242 & 3.0123 \\
\hline Mar-17 & 3.1583 & 3.1319 & Sep-19 & 3.0329 & 3.0052 \\
\hline Apr-17 & 3.1521 & 3.1468 & Oct-19 & 3.0542 & 3.0129 \\
\hline May-17 & 3.0933 & 3.1411 & Nov-19 & 3.0545 & 3.0318 \\
\hline Jun-17 & 3.0909 & 3.0868 & Dec-19 & 2.4573 & 2.7814 \\
\hline
\end{tabular}




\section{Performance Evaluation of Model}

The performance of the proposed model is evaluated by using the Mean Absolute Percentage Error (MAPE) in Eq. (14).

$$
\begin{aligned}
M A P E & =\frac{1}{n} \sum_{t=1}^{n} \frac{\left|Y(t)-F^{\prime}(t)\right|}{Y(t)} \times 100 \% \\
& =\frac{1}{59} \sum_{t=1}^{59} \frac{\left|Y(t)-F^{\prime}(t)\right|}{Y(t)} \times 100 \% \\
& =\frac{1}{59} \times 0.5838 \times 100 \% \\
& =0.9895 \%
\end{aligned}
$$

According to Lewis (1982), the MAPE value below $10 \%$ is considered as highly accurate. The fuzzy time series-Markov chain in exchange rate forecasting obtained a value of $0.9895 \%$. Therefore, the proposed method is highly accurate for exchange rate forecasting in this study.

\section{Comparison of Performance Evaluation}

Exchange rate forecasting between MYR and SGD is performed by using fuzzy time series to provide a comparison model. The actual value and the forecasting value of the exchange rate using fuzzy time series and fuzzy time seriesMarkov chain are depicted in Figure 3.

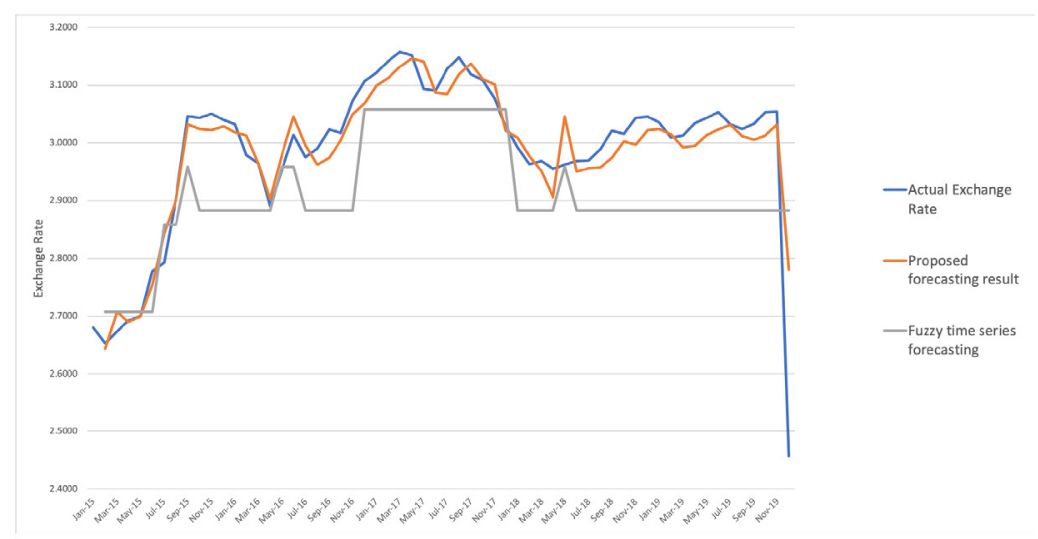

Figure 3: Comparisons in Exchange Rate Forecasting

From Figure 3, the comparison graph shows that the forecasting result of the proposed model is closer to the actual exchange rate.
The MAPE value for fuzzy time series forecasting is also calculated. The result is shown in Table 3.

Table 3: Comparison of MAPE Values

\begin{tabular}{cc}
\hline Forecasting methods & MAPE value \\
\hline Fuzzy Time Series & $3.4306 \%$ \\
Fuzzy Time Series Markov Chain & $0.9895 \%$ \\
\hline
\end{tabular}

\section{Conclusion}

This study explores the benefit of fuzzy time series-Markov chain in the forecasting of exchange rate between Malaysian Ringgit (MYR) and Singapore Dollar (SGD). The results indicated that the integrated model has a better forecasting accuracy compared to the classical models such as fuzzy time series and Markov chain. Both fuzzy time series model and fuzzy time series-Markov chain model give a satisfied performance in the forecasting of exchange rate. However, the fuzzy time series-Markov chain 
has a smaller MAPE value which means higher accuracy in exchange rate forecasting.

For a further study, the advanced software packages such as MATLAB, SAS, and MINITAB can be applied to the proposed model to simulate the results conveniently and effectively. Besides, further exploration such as the use of higherorder fuzzy time series model or higher-order Markov chain model is needed to aim for a better performance in exchange rate forecasting.

\section{Acknowledgements}

This work is a part of the final year project under the BSc of Financial Mathematics program, FTKKI, UMT. The authors are grateful to anonymous referees for comments and suggestions that helped improve the paper.

\section{References}

Adesokan, I., Ngare, P., \& Kilishi , A. (2017). Analyzing expected returns of a stock using the Markov chain model and the capital asset pricing model. Applied Mathematical Sciences, 11(56), 2777-2788.

Adhikari, R., \& Agrawal, R. K. (2013). An introductory study on time series modeling and forecasting. LAP Lambert Academic Publishing, Germany.

Ali, O. A., Ali, A. Y., \& Sumait, B. S. (2015). Comparison between the effects of different different types of membership functions on fuzzy logic controller performance. International Journal of Emerging Engineering Research and Technology, 3 (3), 76-83.

Avrachenkov, K. E., \& Sanchez, E. (2002). Fuzzy Markov Chains and Decision-Making. Fuzzy Optimization and Decision Making, 1, 143-159.

Boiroju, N. K., Rao, M. V., \& Reddy, M. K. (2011). Forecasting Foreign Exchange Rates Using Fuzzy Time Series. International Journal of Statistics and Systems, 6(1), 153161.
Chang, S. S. L., \& Zadeh, L. A. (1972). On fuzzy mapping and control. IEEE Transactions on Systems, Man, and Cybernetics, 2, 30-34.

Chatfield, C. (2000). Time series forecasting. Chapman and Hall, London.

Chen, S. M. (1996). Forecasting enrollments based on fuzzy time series. Fuzzy Sets and Systems, 81, 311-319.

Ching, W. K., \& Ng, M. (2006). Markov chains: Models, algorithms and applications. Springer, New York.

Erdogan, O., \& Goksu, A. (2014). Forecasting Euro and Turkish Lira exchange rates with Artificial Neural Networks (ANN). International Journal of Academic Research in Accounting, Finance and Management Sciences, 4(4), 307-316.

He, Z. C., \& Jiang , W. (2018). A new belief Markov chain model and its application in inventory prediction. International Journal of Production Research, 56(8), 2800-2817.

Islam, M. D., \& Hossain, E. (2020). Foreign Exchange Currency Rate Prediction using a GRU-LSTM Hybrid Network. Soft Computing Letters, 1-20.

Lewis, C. D. (1982). Industrial and business forecasting methods: A practical guide to exponential smoothing and curvefittin. Butterworth Scientific, London.

Li, G., \& Xiu, B. (2014). Fuzzy Markov chains based on the fuzzy transition probability. The 26th Chinese Control and Decision Conference, 4351-4356.

Madi, E., Naim, S., Yaafar, A., Yaakob, A. M., \& Yusoff, B. (2020). Agreement Matrix Based on Fuzzy DecisionMaking to Rank Ship Berthing Criteria. International Journal of Engineering Trends and Technology, 68(12), 31-36.

Meyer, A., \& Zimmermann, H. -J. (2011). Applications of Fuzzy Technology in Business Intelligence. International Journal of Computers, Communications \& Control, 4(3), 428-441. 
Reid, R. D., \& Sanders, N. R. (2010). Operations management an integrated approach, $4^{\text {th }}$ edition. Wiley, Hoboken, New Jersey.

Sahin, A., Kumbasar, T., Yesil, E., Dodurka, M. F., \& Karasakal, O. (2014). An approach to represent time series forecasting via fuzzy numbers. $2^{\text {nd }}$ International Conference on Artificial Intelligence, Modelling and Simulation, 51-56.

Seneta, E. (2006). Markov and the the creation of Markov chains. MAM 2006: Markov Anniversary Meeting. Boson Books, Raleigh, North Carolina, 1-20.

Song, Q., \& Chissom, B. S. (1993). Forecasting enrolments with fuzzy time series-Part I. Fuzzy Sets and Systems, 54(1), 1-9.

Song, Q., \& Chissom, B. S. (1994). Forecasting enrollments with fuzzy time series-Part II. Fuzzy Sets and Systems, 62(1), 1-8.

Tayal, D., Sonawani, S., Ansari, G., \& Gupta, C. (2011). Fuzzy time series forecasting of low dimensional numerical data. International Journal of Engineering Research and Applications, 2(1), 132-135.
Tsaur, R. C. (2012). A fuzzy time series-Markov chain model with an application to forecast the exchange rate between the Taiwan And US Dollar. International Journal of Innovative Computing, Information and Control, 8, 4931-4942.

Uzun, B., \& Kiral, E. (2017). Application of Markov chains-fuzzy states to gold price. Procedia Computer Science, 120, 365-371 .

Zadeh, L. A. (1965). Fuzzy sets. Information and Control, 8, 338-353.

Zadeh, L. A. (1973). Outline of a new approach to the analysis of complex systems and decision processes. IEEE Transactions On Systems, Man, and Cybernetics, Smc-3(1), 28-44.

Zhang, D., \& Zhang X. M. (2009). Study on forecasting the stock market trend based on stochastic analysis method. International Journal of Business and Management, 4(6). 
